ANNALES

POLONICI MATHEMATICI

$87(2005)$

\title{
A note on Bézout's theorem
}

\author{
by SŁawomir Rams, Piotr Tworzewski and \\ TADEusz WiniaRski (Kraków)
}

\begin{abstract}
We present a version of Bézout's theorem basing on the intersection theory in complex analytic geometry. Some applications for products of surfaces and curves are also given.
\end{abstract}

1. Introduction. In the intersection theory in complex analytic geometry constructed in $[\mathrm{T}]$ and completed by $[\mathrm{AR}],\left[\mathrm{R}_{1}\right],\left[\mathrm{R}_{2}\right],\left[\mathrm{R}_{3}\right],\left[\mathrm{R}_{4}\right],\left[\mathrm{R}_{5}\right]$, for a system $X_{1}, \ldots, X_{p}$ of irreducible analytic subsets of a complex manifold $N$, one can define the analytically costructible (upper semicontinuous in the Zariski topology) function

$$
d: N \ni x \mapsto d(x):=d\left(X_{1}, \ldots, X_{p}\right)(x) \in \mathbb{N},
$$

which assigns to each point $x$ in $N$ the multiplicity of intersection of the sets $X_{1}, \ldots, X_{p}$ at the point $x$ (see also [A], [ATW] and [CKT]). By definition, the intersection product of these sets is the unique analytic cycle $X_{1} \bullet \cdots \bullet X_{p}$ defined by the equality

$$
\nu\left(X_{1} \bullet \cdots \bullet X_{p}, x\right)=d\left(X_{1}, \ldots, X_{p}\right)(x) \quad \text { for } x \in N,
$$

where $\nu(C, x)$ denotes the degree of the analytic cycle $C$ at $x$. One can extend this definition in the natural way to the case of analytic cycles. Some details of this construction will be given in the next section of this paper.

One can observe that for a system of analytic cycles $C_{1}, \ldots, C_{p}$ on $N$ and each point $a \in N$ we have "by definition" the Bézout type equality

$$
\nu\left(C_{1} \bullet \cdots \bullet C_{p}, a\right)=d\left(C_{1}, \ldots, C_{p}\right)(a),
$$

saying that the multiplicity of the intersection product of our system coincides with the intersection multiplicity at the point. Usually it is possible to

2000 Mathematics Subject Classification: 13H15, 14B05, 14C17, 32B99.

Key words and phrases: intersection theory, complex analytic geometry, Bézout's theorem, analytic cycle, singularity.

This research was partially supported by KBN Grants: 2P03A01522, 2P03A01625.

The first author was supported by a Fellowship of the Foundation for Polish Science. 
find relations between $d\left(C_{1}, \ldots, C_{p}\right)(a)$ and the product $\nu\left(C_{1}, a\right) \cdots \nu\left(C_{p}, a\right)$ of the multiplicities of the cycles, and so we obtain inequalities or equalities extending the classical Bézout's theorem to the case of analytic sets.

The organization of this paper is as follows. Section 2 is of preparatory nature; we collect together basic constructions and facts on intersection theory in complex analytic geometry. In Section 3 our main results are proved, and then used, in Section 4, to present some applications for products of surfaces and curves. Some comments on the presented version of Bézout's theorem can be found in $\left[\mathrm{N}_{2}\right]$ and $\left[\mathrm{N}_{1}\right]$.

2. Intersection theory. In this paper analytic means complex analytic, and manifold means a second-countable complex manifold. An analytic cycle on a manifold $M$ is a formal sum

$$
C=\sum_{j \in J} \alpha_{j} Y_{j}
$$

where $\alpha_{j} \neq 0$ for $j \in J$ are integers and $\left\{Y_{j}\right\}_{j \in J}$ is a locally finite family of nonempty, pairwise distinct, irreducible analytic subsets of the manifold $M$. The zero cycle $C=0$ is defined by $J=\emptyset$. The family of all analytic cycles on $N$ will be considered with the natural structure of $\mathbb{Z}$-module.

The analytic set $\bigcup_{j \in J} Y_{j}$ is called the support of the cycle $C$. The sets $Y_{j}$ are called the components of $C$ with multiplicities $\alpha_{j}, j \in J$. We say that the cycle $C$ is positive if $\alpha_{j}>0$ for all $j \in J$. If all the components of $C$ have the same dimension $k, C$ will be called a $k$-cycle. For a cycle $C$ and an open subset $V$ of $M$ we can define in the natural way the restriction $C \cap V$ of $C$ to $V$ (cf. [ $\left.\left.\mathrm{R}_{3}\right]\right)$. If $\varphi: M \rightarrow M^{\prime}$ is a biholomorphism of manifolds then we define the image $\varphi(C)$ of $C$ by

$$
\varphi(C)=\sum_{j \in J} \alpha_{j} \varphi\left(Y_{j}\right)
$$

Now, let $M$ be an $m$-dimensional manifold and let $Y$ be a pure $k$-dimensional analytic subset of $M$. For $x \in N$ we denote by $\nu(Y, x)$ the degree of $Y$ at the point $x$ [D, p. 194]. This degree is equal to the classical algebraic Samuel multiplicity, and the so-called Lelong number of $Y$ at $x$. In this paper we will consider a natural extension of this definition to the case of an arbitrary analytic cycle. Namely, if $C=\sum_{j \in J} \alpha_{j} Y_{j}$ is an analytic cycle on $M$, then the sum

$$
\nu(C, x)=\sum_{j \in J} \alpha_{j} \nu\left(Y_{j}, x\right)
$$

is well defined and we call it the degree of the cycle $C$ at the point $x$.

For the cycle $C$ there exists a unique decomposition

$$
C=C_{(m)}+C_{(m-1)}+\cdots+C_{(0)},
$$


where $C_{(j)}$ is a $j$-cycle for $j=0, \ldots, m$. We define the extended degree of $C$ at $x$ by the formula

$$
\widetilde{\nu}(C, x)=\left(\nu\left(C_{(m)}, x\right), \ldots, \nu\left(C_{(0)}, x\right)\right) \in \mathbb{Z}^{m+1} .
$$

Denote by $\nu(C)$ and $\widetilde{\nu}(C)$ the functions

$$
\nu(C): M \ni x \mapsto \nu(C, x) \in \mathbb{Z}, \quad \widetilde{\nu}(C): M \ni x \mapsto \widetilde{\nu}(C, x) \in \mathbb{Z}^{m+1} .
$$

Observe that $\nu(C, x)=|\widetilde{\nu}(C, x)|$, where $|\mu|$ denotes the sum of the coordinates of $\mu \in \mathbb{Z}^{m+1}$.

Let $M$ be an $m$-dimensional manifold and let $S$ be a closed $s$-dimensional submanifold of $M$. For an arbitrary analytic cycle $C=\sum_{j \in J} \alpha_{j} Y_{j}$ in $M$ the part of $C$ supported by $S$ is defined as

$$
C^{S}=\sum_{j \in J, Y_{j} \subset S} \alpha_{j} Y_{j}
$$

Observe that every analytic cycle has the decomposition $C=C^{S}+\left(C-C^{S}\right)$. If $C$ is positive, then so are both parts of this decomposition.

Let $U$ be an open subset of $M$ such that $U \cap S \neq \emptyset$. Denote by $\mathcal{H}(U)$ the set of all $\mathcal{H}:=\left(H_{1}, \ldots, H_{m-s}\right)$ satisfying the following conditions:

(1) $H_{j}$ is a smooth hypersurface in $U$ containing $U \cap S$ for $j=1, \ldots, m-s$,

(2) $\bigcap_{j=1}^{m-s} T_{x}\left(H_{j}\right)=T_{x} S$ for each $x \in U \cap S$.

For a given analytic subset $Z$ of $M$ of pure dimension $k$ we denote by $\mathcal{H}(U, Z)$ the set of all $\mathcal{H} \in \mathcal{H}(U)$ such that $((U \backslash S) \cap Z) \cap H_{1} \cap \cdots \cap H_{j}$ is an analytic subset of $U \backslash S$ of pure dimension $k-j$ (or empty) for $j=1, \ldots, m-s$.

For every $\mathcal{H}=\left(H_{1}, \ldots, H_{m-s}\right) \in \mathcal{H}(U, Z)$ an analytic cycle $Z \cdot \mathcal{H}$ in $S \cap U$ is defined by the following algorithm (cf. [T], see also [F]), where in each step we have only proper intersections, and so the intersection product is given by the classical theory (cf. $\left.[\mathrm{D}],\left[\mathrm{W}_{1}\right]\right)$. For $\mathcal{H}$ define

$$
d:=\min \left\{j \in\{0,1, \ldots, m-s\}:((U \backslash S) \cap Z) \cap \bigcap_{i=1}^{j} H_{i}=\emptyset\right\},
$$

and consider the following

Algorithm 1.

Step 0: Let $Z_{0}=Z \cap U$. Then $Z_{0}=Z_{0}^{S \cap U}+\left(Z_{0}-Z_{0}^{S \cap U}\right)$.

Step 1: Let $Z_{1}=\left(Z_{0}-Z_{0}^{S \cap U}\right) \cdot H_{1}$ and $Z_{1}=Z_{1}^{S \cap U}+\left(Z_{1}-Z_{1}^{S \cap U}\right)$.

Step 2: Let $Z_{2}=\left(Z_{1}-Z_{1}^{S \cap U}\right) \cdot H_{2}$ and $Z_{2}=Z_{2}^{S \cap U}+\left(Z_{2}-Z_{2}^{S \cap U}\right)$.

Step $d$ : Let $Z_{d}=\left(Z_{d-1}-Z_{d-1}^{S \cap U}\right) \cdot H_{d}$. We have $Z_{d}=Z_{d}^{S \cap U}$.

We call a positive analytic cycle $Z \cdot \mathcal{H}=Z_{0}^{S \cap U}+Z_{1}^{S \cap U}+\cdots+Z_{d}^{S \cap U}$ in $S \cap U$ the result of the above algorithm. 
Definition 2. For $c \in S$ we call

$$
\widetilde{g}(c)=\widetilde{g}(Z, S)(c):=\min _{\operatorname{lex}}\{\widetilde{\nu}(Z \cdot \mathcal{H}, c): \mathcal{H} \in \mathcal{H}(U, Z), c \in U\} \in \mathbb{N}^{s+1}
$$

the extended index of intersection and $g(c)=|\widetilde{g}(c)|$ the index of intersection of $Z$ with the submanifold $S$ at the point $c$.

For a system of irreducible analytic sets $X_{1}, \ldots, X_{p}$ on a complex manifold $N$ we can consider the analytic set $Z=X_{1} \times \cdots \times X_{p}$ in $M=N^{p}$ and $S=\Delta_{N}$ the diagonal of $N^{p}$. By [T, Thm. 6.2] the function

$d: N \ni x \mapsto d(x):=d\left(X_{1}, \ldots, X_{p}\right)(x)=g\left(X_{1} \times \cdots \times X_{p}, \Delta_{N}\right)(x, \ldots, x) \in \mathbb{N}$ is analytically costructible and by [T, Prop. 2.1] we can state the following

Definition 3. The intersection product of the system $X_{1}, \ldots, X_{p}$ is the unique analytic cycle $X_{1} \bullet \cdots \bullet X_{p}$ such that $\nu\left(X_{1} \bullet \cdots \bullet X_{p}, x\right)=d(x)$ for $x \in N$.

One can extend this definition in the natural way to the case of analytic cycles (see [T, Def. 6.4]). Namely, let

$$
C_{k}=\sum_{j_{k} \in J_{k}} \alpha_{j_{k}}^{(k)} X_{j_{k}}^{(k)}, \quad k=1, \ldots, p,
$$

be analytic cycles on a manifold $N$.

Definition 4 . The intersection product of the cycles $C_{1}, \ldots, C_{p}$ is defined by

$$
C_{1} \bullet \cdots \bullet C_{p}=\sum_{j_{k} \in J_{k}, k=1, \ldots, p} \alpha_{j_{1}}^{(1)} \cdots \alpha_{j_{p}}^{(p)} X_{j_{1}}^{(1)} \bullet \cdots \bullet X_{j_{p}}^{(p)} .
$$

We conclude this section with a useful theorem which follows directly from our definitions and [AR, Cor. 5].

THEOREM 5. If $C_{1}, \ldots, C_{p}$ is a system of cycles on a complex manifold $N, V$ is an open subset of $N$ and $\varphi: V \rightarrow N^{\prime}$ is a biholomorphism, then

$$
\varphi\left(C_{1} \cap V\right) \bullet \cdots \bullet \varphi\left(C_{p} \cap V\right)=\varphi\left(\left(C_{1} \bullet \cdots \bullet C_{p}\right) \cap V\right) .
$$

3. Bézout's theorem. In this section an analytic cycle $A=\sum_{j \in J} \alpha_{j} X_{j}$ in $\mathbb{C}^{n}$ is called homogeneous (resp. projective) if all sets $X_{j}$ are cones in $\mathbb{C}^{n}$ (resp. projective varieties).

TheOREM 6. Let $C_{1}, \ldots, C_{p}$ be homogeneous cycles. Then the cycle $C_{1} \bullet \cdots \bullet C_{p}$ is homogeneous and

$$
\operatorname{deg}\left(C_{1} \bullet \cdots \bullet C_{p}\right)=\operatorname{deg} C_{1} \cdots \operatorname{deg} C_{p} .
$$

Proof. We maintain the notation of the previous section. It suffices to prove the theorem for irreducible cones $X_{1}, \ldots, X_{p}$. Observe that for $Z=$ $X_{1} \times \cdots \times X_{p}$

$$
d\left(X_{1}, \ldots, X_{p}\right)(0)=g\left(Z, \Delta_{\mathbb{C}^{n}}\right)(0, \ldots, 0)=\left|\widetilde{g}\left(Z, \Delta_{\mathbb{C}^{n}}\right)(0)\right|
$$


By [AR, Cor. 3] there exists a system $\mathcal{H}=\left(H_{1}, \ldots, H_{m-s}\right)$ of hyperplanes such that $\widetilde{g}\left(Z, \Delta_{\mathbb{C}^{n}}\right)(0)=\widetilde{\nu}(Z \cdot \mathcal{H}, 0)$. The classical Bézout's theorem used in each step of Algorithm 1 gives

$$
\left|\widetilde{g}\left(Z, \Delta_{\mathbb{C}^{n}}\right)(0)\right|=\nu(Z, 0)=\operatorname{deg} X_{1} \cdots \operatorname{deg} X_{p} .
$$

Then we have the equality

$$
\nu\left(X_{1} \bullet \cdots \bullet X_{p}, 0\right)=d\left(X_{1}, \ldots, X_{p}\right)(0)=\operatorname{deg} X_{1} \cdots \operatorname{deg} X_{p} .
$$

It is easy to check that $X_{1} \bullet \cdots \bullet X_{p}$ is homogeneous, so

$$
\nu\left(X_{1} \bullet \cdots \bullet X_{p}, 0\right)=\operatorname{deg} X_{1} \bullet \cdots \bullet X_{p}
$$

and the theorem follows.

For a projective cycle $C=\sum_{j \in J} \alpha_{j} Y_{j}$ we define the cycle $\widehat{C}:=\sum_{j \in J} \alpha_{j} \widehat{Y}_{j}$, where $\widehat{Y}_{j}$ denotes the cone over the variety $Y_{j}$.

In the case of the analytic intersection product we have the following version of Bézout's theorem.

Theorem 7. Let $C_{1}, \ldots, C_{p}$ be projective cycles and let $\alpha_{0}$ be the multiplicity of the point $\{0\}$ in the intersection product $\widehat{C}_{1} \bullet \cdots \bullet \widehat{C}_{p}$. Then

$$
\operatorname{deg}\left(C_{1} \bullet \cdots \bullet C_{p}\right)=\operatorname{deg} C_{1} \cdots \operatorname{deg} C_{p}-\alpha_{0} .
$$

Proof. Since the intersection product is linear (see [AR, Cor. 5]), we can assume that $C_{1}, \ldots, C_{p}$ are varieties of dimensions $d_{1}, \ldots, d_{p}$ respectively.

We choose a point $Q:=\left(1: q_{1}: \ldots: q_{n}\right) \in C_{1} \cap \ldots \cap C_{p}$. To simplify our notation we put $V:=\left(\{1\} \times \mathbb{C}^{n}\right)^{p} \subset \mathbb{C}^{p(n+1)}$ and $\mathcal{Q}:=\left(1, q_{1}, \ldots, q_{n}\right)$. The Grassmannian of affine $r$-planes through the point $\mathcal{Q}$ is denoted by $\mathrm{G}_{\mathcal{Q}}^{r}\left(\mathbb{C}^{p(n+1)}\right)$.

By [AR, Cor. 3] the set of affine hyperplanes $H$ containing $\mathcal{Q}$, meeting the space $V$ properly and satisfying the condition

$$
\nu\left(\left(\prod_{i=1}^{p} \widehat{C}_{i} \cdot H\right)^{\Delta}, Q\right)=\left(\widetilde{g}\left(\prod_{i=1}^{p} \widehat{C}_{i}, \Delta\right)(Q)\right)_{1},
$$

where $(v)_{1}$ stands for the first coordinate of a vector $v$, is an open and dense subset of $\mathrm{G}_{\mathcal{Q}}^{p(n+1)-1}\left(\mathbb{C}^{p(n+1)}\right)$. Since the map

$$
H \mapsto H \cap V \in \mathrm{G}_{\mathcal{Q}}^{p(n+1)-2}(V)
$$

restricted to the set of hyperplanes that do not contain $V$ is continuous, we can assume that

$$
\nu\left(\left(\prod_{i=1}^{p} C_{i} \cdot(H \cap V)\right)^{\Delta}, \mathcal{Q}\right)=\left(\widetilde{g}\left(\prod_{i=1}^{p} C_{i}, \Delta\right), Q\right)_{1} .
$$


Proceeding inductively we construct a system $\mathcal{H}=\left(H_{1}, \ldots, H_{p n-\sum d_{i}}\right)$ of affine hypersurfaces such that

$$
\begin{aligned}
\widetilde{\nu}\left(\prod \widehat{C}_{i} \cdot \mathcal{H}, \mathcal{Q}\right) & =\widetilde{g}\left(\prod \widehat{C}_{i}, \Delta\right)(\mathcal{Q}), \\
\widetilde{\nu}\left(\left.\prod C_{i} \cdot \mathcal{H}\right|_{V}, \mathcal{Q}\right) & =\widetilde{g}\left(\prod C_{i}, \Delta\right)(Q),
\end{aligned}
$$

where $\left.\mathcal{H}\right|_{V}:=\left(H_{1} \cap V, \ldots, H_{p n-\sum d_{i}} \cap V\right)$. Here, by abuse of notation, both the diagonal in $\left(\mathbb{C}^{n}\right)^{p}$ and the one in $\left(\mathbb{P}^{n}\right)^{p}$ are denoted by $\Delta$. Moreover, we identify $Q$, resp. $\mathcal{Q}$, with the corresponding point in $\Delta$.

Applying [TW, Thm. 2.2] in each step of Algorithm 1 we get

$$
\widetilde{\nu}\left(\prod \widehat{C}_{i} \cdot \mathcal{H}, \mathcal{Q}\right)=\widetilde{\nu}\left(\left.\prod C_{i} \cdot \mathcal{H}\right|_{V}, \mathcal{Q}\right)
$$

which implies

$$
\widetilde{g}\left(\prod \widehat{C}_{i}, \Delta\right)(\mathcal{Q})=\widetilde{g}\left(\prod C_{i}, \Delta\right)(Q) .
$$

As an immediate consequence we obtain the equality

$$
\widehat{C}_{1} \bullet \cdots \bullet \widehat{C}_{p}=\sum_{j \geq 1} \alpha_{j} \widehat{Y}_{j}+\alpha_{0} \cdot\{0\},
$$

where $C_{1} \bullet \cdots \bullet C_{p}:=\sum_{j \geq 1} \alpha_{j} Y_{j}$. By Theorem 6, we have

$$
\operatorname{deg}\left(C_{1} \bullet \cdots \bullet C_{p}\right)=\operatorname{deg} C_{1} \cdots \operatorname{deg} C_{p}-\alpha_{0} \text {. }
$$

4. Applications. To show an application of Theorem 6 we analyze the product of an algebraic surface and a curve. We start with the following lemma:

Lemma 8. Let $S$ be a projective surface and let $C \subset S$ be a curve that has no common component with $\operatorname{Sing}(S)$. Then

$$
\widehat{S} \bullet \widehat{C}=\widehat{C}+\sum_{i=1}^{r} \alpha_{i} \widehat{a}_{i}+\alpha \cdot\{0\},
$$

where

(1) $a_{i} \in \operatorname{Sing}(S) \cup \operatorname{Sing}(C)$ for $i=1, \ldots, r$,

(2) $\alpha_{i} \geq\left(\nu\left(S, a_{i}\right)-1\right) \nu\left(C, a_{i}\right)$ for $i=1, \ldots, r$,

(3) $\alpha \geq 0$.

Proof. (1) It is obvious that all components of the cycle $\widehat{S} \bullet \widehat{C}$ are cones. If $a \in \operatorname{Reg}(S) \cap \operatorname{Reg}(C)$, then the germ $\widehat{C}_{a}$ can be cut out from $\widehat{S}_{a}$ by one smooth hypersurface that meets $\widehat{S}_{a}$ transversally along $\widehat{C}_{a}$. The latter implies that $\widehat{C}$ appears in the intersection product with multiplicity one and (1) holds. 
(2) Since $g(\widehat{S} \times \widehat{C}, \Delta)\left(1, a_{i}, 1, a_{i}\right)=\nu\left(C, a_{i}\right)+\alpha_{i}$, it suffices to prove the inequality

$$
g(\widehat{S} \times \widehat{C}, \Delta)\left(1, a_{i}, 1, a_{i}\right) \geq \nu\left(S, a_{i}\right) \cdot \nu\left(C, a_{i}\right) .
$$

The latter is an immediate consequence of $\left[\mathrm{W}_{2}\right.$, Property 3].

(3) To prove that $\alpha \geq 0$ choose $\mathcal{H}:=\left(H_{1}, \ldots\right) \in \mathcal{H}(U, \widehat{S} \times \widehat{C})$, where $U$ is a neighbourhood of 0 , such that

$$
\widetilde{\nu}((\widehat{S} \times \widehat{C}) \cdot \mathcal{H}, 0)=\widetilde{g}(\widehat{S} \times \widehat{C}, \Delta)(0) .
$$

By [AR, Corollary 3] we can assume $H_{i}$ to be hyperplanes. Let $Z_{i}, Z_{i}^{\Delta}$ denote the results of the $i$ th step of Algorithm 1 applied to $\mathcal{H}, \widehat{S} \times \widehat{C}, \Delta$, where $\Delta$ is the diagonal in $\left(\mathbb{C}^{n+1}\right)^{2}$.

One can see that $Z_{1}^{\Delta}=Z_{2}^{\Delta}=0$. Thus

$$
Z_{3}=\left(H_{1} \cap H_{2} \cap H_{3}\right) \cdot(\widehat{S} \times \widehat{C})=j(\widehat{C})+\sum \beta_{k} C_{k},
$$

where $j: \mathbb{C}^{n+1} \ni x \mapsto(x, x) \in \Delta$ and $C_{k}$ are two-dimensional cones.

Observe that $j\left(\widehat{a}_{i}\right) \subset \bigcup C_{k}$ for each $a_{i}$ such that $\alpha_{i}>0$. Now

$$
Z_{4}=H_{4} \cdot\left(\sum \beta_{k} C_{k}\right)=\sum \beta_{k}^{\prime} l_{k}
$$

where $l_{k}$ are lines. Consequently

$$
\widetilde{g}(\widehat{S} \times \widehat{C}, \Delta)(0)=\left(\operatorname{deg}(C), \sum_{l_{k} \subset \Delta} \beta_{k}^{\prime}, \sum_{l_{k} \not \subset \Delta} \beta_{k}^{\prime}\right) .
$$

Choose $b \in \widehat{a}_{i} \cap U$. Observe that if $\widehat{a}_{i} \neq l_{k}$ for all $k$, then $\alpha_{i}=0$. Indeed, we have $\mathcal{H} \in \mathcal{H}(U, \widehat{S} \times \widehat{C})$, which yields the equality $g(b)=\nu(\widehat{C}, b)$ and implies that $\widehat{C}$ is the only component of the cycle $\widehat{S} \bullet \widehat{C}$ passing through $b$.

If $\alpha_{i}>0$ then $\widehat{a}_{i}$ is one of the lines $l_{k}$, say $\widehat{a}_{i}=l_{k_{0}}$, and

$$
\widetilde{g}(\widehat{S} \times \widehat{C}, \Delta)(b) \leq_{\operatorname{lex}}\left(\nu(\widehat{C}, b), \beta_{k_{0}}^{\prime}, 0\right) .
$$

Since $(\widetilde{g}(\widehat{S} \times \widehat{C}, \Delta)(b))_{1} \geq \nu(\widehat{C}, b)$, we get $\alpha_{i} \leq \beta_{k_{0}}^{\prime}$ and

$$
\alpha=g(\widehat{S} \times \widehat{C}, \Delta)(0)-\nu\left(\widehat{C}+\sum \alpha_{i} \widehat{a}_{i}, 0\right) \geq \sum_{l_{k} \not \subset \Delta} \beta_{k}^{\prime} \geq 0 .
$$

Now we can state

Corollary 9. Let $S$ be a projective surface and let $C \subset S$ be a curve that has no common component with $\operatorname{Sing}(S)$. Then

$$
\sum_{a \in \operatorname{Sing}(S) \cap C}(\nu(S, a)-1) \cdot \nu(C, a) \leq(\operatorname{deg}(S)-1) \cdot \operatorname{deg}(C) .
$$

Proof. We maintain the notation of Lemma 8. By Theorem 6 we have

$$
\operatorname{deg}(\widehat{S} \bullet \widehat{C})=\operatorname{deg}(S) \cdot \operatorname{deg}(C),
$$


so Lemma 8 yields

$$
\operatorname{deg}(S) \cdot \operatorname{deg}(C)=\operatorname{deg}(C)+\sum \alpha_{i}+\alpha
$$

By Lemma 8(2) we get

$$
\sum_{a \in \operatorname{Sing}(S) \cap C}(\nu(S, a)-1) \cdot \nu(C, a) \leq(\operatorname{deg}(S)-1) \cdot \operatorname{deg}(C) .
$$

REMARK 10. It is natural to ask to what extent the bounds from Lemma 8 and Corollary 9 are sharp. This amounts to the question how to control the behaviour of the multiplicity of the point 0 in the intersection product of cones. Let us point out that the latter can be expressed with the help of the Hilbert function (see $[\mathrm{AR}],[\mathrm{AM}]$ ) and computed using various computer algebra systems.

\section{References}

[AM] R. Achilles and M. Manaresi, Multiplicities of bigraded rings and intersection theory, Math. Ann. 309 (1997), 573-591.

[A] M. Montserrat Alonso Ferrero, On the index of contact, Ann. Polon. Math. 83 (2004), 77-86.

[AR] R. Achilles and S. Rams, Intersection numbers, Segre numbers and generalized Samuel multiplicities, Arch. Math. (Basel) 77 (2001), 391-398.

[ATW] R. Achilles, P. Tworzewski and T. Winiarski, On improper isolated intersection in complex analytic geometry, Ann. Polon. Math. 51 (1990), 21-36.

[CKT] E. Cygan, T. Krasiński and P. Tworzewski, Separation of algebraic sets and Eojasiewicz exponent of polynomial mappings, Invent. Math. 136 (1999), 75-87.

[D] R. Draper, Intersection theory in analytic geometry, Math. Ann. 180 (1969), 175-204.

[F] W. Fulton, Intersection Theory, Springer, Berlin, 1984.

$\left[\mathrm{N}_{1}\right] \quad$ K. J. Nowak, Improper intersections in complex analytic geometry, Dissertationes Math. 391 (2001), 1-58.

$\left[\mathrm{N}_{2}\right] \quad-$, Supplement to the paper "Improper intersections in complex analytic geometry", Ann. Polon. Math. 76 (2001), 303.

[R $\left.\mathrm{R}_{1}\right] \quad$ S. Rams, Convergence of holomorphic chains, ibid. 65 (1997), 227-234.

$\left[\mathrm{R}_{2}\right] \quad-$, Bézout-type theorems for certain analytic sets, Bull. Polish Acad. Sci. Math. 46 (1998), 277-283.

$\left[\mathrm{R}_{3}\right] \quad-$, On the intersection product of analytic cycles, Ann. Polon. Math. 73 (2000), $135-146$.

$\left[\mathrm{R}_{4}\right] \quad-$, On intersections with hypersurfaces and normal pseudo-flatness, Bull. Polish Acad. Sci. Math. 48 (2000), 335-340.

$\left[\mathrm{R}_{5}\right] \quad-$, On the index of contact and multiplicities for bigraded ring, Manuscripta Math. 106 (2001), 339-347.

[T] P. Tworzewski, Intersection theory in complex analytic geometry, Ann. Polon. Math. 62 (1995), 177-191.

[TW] P. Tworzewski and T. Winiarski, Cycles of zeroes of holomorphic mappings, Bull. Polish Acad. Sci. Math. 37 (1989), 95-101. 
[V] W. Vogel, Results on Bézout's Theorem, Tata Lecture Notes 74, Springer, Berlin, 1984.

[W $\mathrm{W}_{1}$ T. Winiarski, Continuity of total number of intersection, Ann. Polon. Math. 47 (1986), 155-178.

$\left[\mathrm{W}_{2}\right] \quad-$ Local properties of intersection, in: Deformations of Mathematical Structures, Kluwer, 1989, 141-150.

S. Rams and P. Tworzewski

T. Winiarski Institute of Mathematics Jagiellonian University Reymonta 4 30-059 Kraków, Poland

E-mail: slawomir.rams@im.uj.edu.pl piotr.tworzewski@im.uj.edu.pl

\author{
Institute of Mathematics \\ Pedagogical University of Cracow \\ Podchorążych 2 \\ 30-084 Kraków, Poland \\ E-mail: twiniarski@autocom.pl
}

\author{
Reçu par la Rédaction le 30.11.2004 \\ Révisé le 1.4.2005
}

